\title{
Europäische versus nationale Gleichheit
}

\author{
Jürgen Gerhards
}

Berliner Studien zur Soziologie Europas

Nr. 2

Oktober 2005

Freie Universität Berlin, Institut für Soziologie, Garystraße 55, D-14195 Berlin 
Die „Berliner Studien zur Soziologie Europas“ des Lehrstuhls für Makrosoziologie der Freien Universität Berlin verstehen sich als ein Ort zur Vorpublikation von Beiträgen, die später in Fachzeitschriften und Sammelbänden veröffentlicht werden sollen. Die Beiträge sollen helfen, eine Soziologie Europas zu profilieren; sie stehen auch im Kontext des Master-Studiengangs „Soziologie - Europäische Gesellschaften“.

Gegenstand der Reihe sind Beiträge zur Analyse der Herausbildung einer europäischen Gesellschaftsstruktur und -kultur, vergleichende Analysen, die die Unterschiede und Gemeinsamkeiten zwischen verschiedenen europäischen Gesellschaften thematisieren, sowie theoretische Versuche einer Soziologie Europas.

Ziel der Reihe ist es, durch die frühe Verbreitung dieser Arbeiten den wissenschaftlichen Gedankenaustausch zu fördern. Die Beiträge sind nur über das Internet als pdf-Datei zu beziehen.

Zitationsweise: BSSE-Arbeitspapier Nr.2. Berlin: Freie Universität Berlin.

Dieser Artikel erschien auch in: Martin Heidenreich (Hg.): Die Europäisierung sozialer Ungleichheit. Zur transnationalen Klassen- und Sozialstrukturanalyse Frankfurt: Campus (2006) S. 253-278. 
Der europäische Integrationsprozess lässt sich als ein Prozess der Verlagerung von Souveränitätsrechten von den Nationalstaaten auf die Institutionen der Europäischen Union interpretieren. Die heutige Europäische Union startete mit der Festlegung einer gemeinsamen Verwaltung für die Kohle- und Stahlindustrie. Schritt für Schritt wurden andere Bereiche in den Prozess der Vertiefung einbezogen: Eine Zollunion wurde gegründet, ein gemeinsamer Binnenmarkt und eine Wirtschafts- und Währungsunion wurden gebildet, und schließlich wurde für eine Teilgruppe der EULänder eine gemeinsame Währung eingeführt. ${ }^{1}$

Der Prozess der zunehmenden europäischen Integration lässt sich aber nicht nur als ein Prozess der Zunahme europäischer Regelungen und der Ausbildung eines eigenständigen europäischen Institutionensystems beschreiben, sondern auch als ein Prozess der Etablierung einer europäischen Werteordnung. Die Union ist auch ein politischer Werteunternehmer, der mit seinen Politiken in die Werteordnungen seiner Mitgliedsländer eingreift. Sie verfügt über ein Skript einer europäischen Gesellschaft und versucht, mit ihren Politiken ihre Vorstellungen einer europäischen Gesellschaft zu realisieren. Dabei hat die EU zum Teil sehr dezidierte Vorstellungen darüber, wie eine Familie, die Ökonomie, der Wohlfahrtsstaat oder eine Zivilgesellschaft idealiter aussehen soll. Wir haben an anderer Stelle mithilfe einer Analyse des Primär- und Sekundärrechts der EU zu zeigen versucht, welche Werteordnung - differenziert nach Wertsphären - die EU präferiert (vgl. Gerhards/Hölscher 2005) ${ }^{2}$. Wir knüpfen in der folgenden Untersuchung an diese Analysen an, konzentrieren uns hier auf das Gleichheitsskript der Europäischen Union und gehen dabei folgenden drei - miteinander verknüpften - Fragen nach.

1. In einem ersten Schritt beschreiben wir mit Rekurs auf das EU-Recht und die EU- Politiken, wie die Europäischen Union die Idee einer allein binnennationalen Gleichheit transnationalisiert und ersetzt hat durch die Idee einer Gleichheit aller Bürger Europas. Ein Blick in die Vertragsentwicklungen wird zeigen, dass die Union Schritt für Schritt das Prinzip der Gleichheit aller Unionsbürger auf unterschiedliche Politikfelder ausgedehnt und damit die Legitimität nationalstaatlicher Schließung und von nationalen Sonderregelungen durchbrochen hat. Dies gilt vor allem für den Bereich der Wirtschaft und die Herstellung eines gemeinsamen europäischen Binnenmarktes.

1 Die Verlagerung von Souveränitätsrechten von den Nationalstaaten auf die EU manifestiert sich in einer Vielzahl von Indikatoren und ist von Politikwissenschaftlern ausführlich beschrieben worden. Die Summe von Entscheidungen seitens des Europäischen Rates bzw. der Europäischen Kommission ist kontinuierlich gestiegen, die Anzahl der Fachministerräte ebenfalls, die Verflechtung zwischen europäischer und nationaler Politik hat zugenommen (Knill 2001), und auch die intermediären Organisationen und Interessengruppen richten ihr Augenmerk zunehmend auf die europäische Ebene (Fligstein/Stone Sweet 2002; Stone Sweet u.a. 2001; Wessels 1997: 283).

2 Ich bedanke mich bei Holger Lengfeld und Mike Schäfer für kritische Kommentare, bei David Glowsky für die Hilfe bei der Rekonstruktion des EU-Skripts, und vor allem bei Silke Hans für die Unterstützung bei den empirischen Analysen. 
2. Manche Autoren gehen davon aus, dass sich mit einer Europäisierung der Politikfelder und einer Strukturierung eines europäischen gesellschaftlichen Raumes auch die Wahrnehmungen der Bürger verändern, so dass man von einer Europäisierung von Einstellungen und Werteorientierungen sprechen kann. Ulrich Beck und Edgar Grande haben jüngst die These formuliert: »Im Anschluss daran soll hier die These entwickelt werden, dass sich die bisherigen Verzerrungen in der Wahrnehmung sozialer Ungleichheiten - das heißt die Herstellung und Aufrechterhaltung der Unvergleichbarkeit von gleichen Ungleichheiten zwischen und über nationale Grenzen hinweg - im Zuge der grenzverändernden Grenzenpolitik der Europäisierung auflösen.« (Beck/Grande 2004: 266) Ob dies wirklich der Fall ist, wollen wir für den Bereich der Gleichheit auf dem Arbeitsmarkt prüfen. In welchem Maße unterstützen die Bürger die Vorstellung, dass ausländische Bürger auf dem Arbeitsmarkt die gleichen Zugangsmöglichkeiten und Rechte genießen wie die eigenen Bürger, oder umgekehrt formuliert: In welchem Maße gehen die Bürger davon aus, dass Inländer gegenüber europäischen Ausländern einen bevorzugten Zugang zu Arbeitsplätzen haben sollen? Unterstützen die EU-Bürger das Skript der EU-Institutionen von einer Gleichheit der Arbeitskräfte oder favorisieren sie ein Ungleichheitskonzept, das zwischen Inländern und Ausländern unterscheidet? Wird die institutionelle Europäisierung also durch eine mentalitätsmäßige Europäisierung (Heidenreich in diesem Band) unterstützt?

Die Frage nach einer Unterstützung des EU-Skripts durch die Bürger ist für die Legitimität der Politiken der EU nicht unerheblich, wie die Ablehnung der europäischen Verfassung in den Mitgliedsländern Frankreich und den Niederlanden gerade gezeigt haben. Demokratien sind strukturell auf die Unterstützung ihrer Bürger angewiesen. Bleibt diese aus, kann es zu Legitimitätsproblemen der Institutionen selbst kommen.

3. Die deskriptiven Befunde werden zeigen, dass das Gleichheitsskript der EU bei den Bürgern Europas keine mehrheitliche Unterstützung findet, wobei es erhebliche Unterschiede zwischen den Ländern gibt. Neben einer Deskription gehen wir in einem dritten Schritt der Frage nach, wie man die gefundenen Unterschiede zwischen den Ländern erklären kann. Dazu formulieren wir zuerst Hypothesen, die wir mit Hilfe einer multivariaten Analyse überprüfen werden. In einem abschließenden Kapitel gehen wir auf mögliche politische Implikationen der Befunde ein. Die empirische Grundlage unserer Rekonstruktion der Werteorientierung der Bürger bilden Sekundäranalysen von repräsentativen Bevölkerungsbefragungen, die in den Mitglieds- und Beitrittsländern durchgeführt und in denen die Bürger nach Werteeinstellungen gefragt wurden.

\section{Das EU-Skript einer Europäisierung der Gleichheit}

Auch wenn der Nationalstaat eine historisch relativ spät entstandene Form der Gesellschaftsformation darstellt, besteht unter Sozialwissenschaftlern wenig Zweifel daran, dass man europäische Gesellschaften des 19. und 20. Jahrhunderts als natio- 
nalstaatlich verfasste Gesellschaften begreifen kann. Die Monopolisierung der Gewalt in der Hand des Staates zur Sicherung nationalstaatlicher Außengrenzen einerseits und zur Durchdringung des inneren Raumes andererseits bildet dabei das zentrale Strukturmerkmal von Nationalstaaten. Militärische Grenzsicherung, Zollpolitik und Einwanderungspolitik bilden die wesentlichen Bereiche der Abgrenzung der Nationalstaaten nach außen. Die Strukturierung des inneren Territoriums erfolgt durch polizeiliche und bürokratische Durchdringung und den Aufbau eines nationalstaatlich begrenzten Institutionengefüges. Dieses reicht vom Bildungssystem, dem Sozialversicherungs- und Gesundheitswesen, über die Ausbildung eines nationalstaatlich begrenzten politischen Systems bis hin zur Konstituierung von nationalen Volkswirtschaften. Komplementär zur nationalstaatlichen Institutionenbildung erfolgt die Inklusion der Menschen, die innerhalb des Territoriums eines Staates leben. Sie werden zu Bürgern ihres Staates, müssen sich erziehen lassen (Schulpflicht), erhalten als Staatsbürger das Recht, ihre Regierung zu wählen (Wahlrecht) und können die wohlfahrtstaatlichen Leistungen ihres und nur ihres Landes in Anspruch nehmen.

Mit der Ausbildung, Demokratisierung und wohlfahrtsstaatlichen Erweiterung der Nationalstaaten ist eine sehr spezifische Codierung der Gleichheitsidee verbunden. Diese bezieht sich dominant und in erster Linie auf die Chancengleichheit aller innerhalb eines Nationalstaats lebenden Bürger, schließt damit die Nichtmitglieder (Mitglieder anderer Nationalstaaten also) weitgehend aus und behandelt diese als ungleich (sieht man von einigen allgemeinen Rechten ab, die für alle Menschen gelten und in den Menschenrechten kodifiziert sind). Ausländer haben in aller Regel nicht das Recht, sich in einem fremden Land niederzulassen, dort zu arbeiten, eine Ausbildung zu genießen, zur Wahl zu gehen oder am Wohlfahrtsstaat zu partizipieren.

Die Soziologie als Wissenschaft der Gesellschaft im Allgemeinen und die Soziologie der Ungleichheit im Besonderen hat sich in ihren Kategoriensystemen dieser Realität des 19. und 20. Jahrhunderts insofern angepasst, als sie Ungleichheiten in erster Linie als binnennationalstaatliche Ungleichheiten (Klassen, Schichten) analysiert, Ungleichheiten zwischen Ländern hingegen systematisch vernachlässigt hat. Dies gilt auch für die ländervergleichende Umfrageforschung. Der Bezugspunkt der Bewertung von Ungleichheiten, Umverteilungspolitiken oder wohlfahrtsstaatlichen Arrangements ist immer die Legitimität einer binnennationalstaatlichen Verteilung von Ressourcen (vgl. z.B. Svallfors 1997; Roller 2000; Mau 2003). Diese verzerrte Perspektive ist jüngst von Ulrich Beck (vgl. z.B. Beck/Grande 2004) in Aufnahme einer Begrifflichkeit von Anthony D. Smith (1983) als methodologischer Nationalismus beschrieben und kritisiert worden.

Der Prozess der politischen Integration Europas ist nun auch mit intendierten Folgen für die Gleichheitsidee verbunden. Die Idee nationalstaatlich begrenzter Gleichheit wird ausgedehnt und europäisiert, eine nationalstaatlich begrenzte Codierung von Gleichheit wird ersetzt durch eine europäisch begrenzte Idee der Gleichheit, die alle Bürger Europas als Gleiche betrachtet und die legitimierte Ungleichheitsgrenze nach außen verschiebt. Natürlich gilt dies vorerst nur für manche gesellschaftliche 
Bereiche und ist zum Teil auch nur schwach entwickelt. Das Wahlrecht der europäischen Ausländer beschränkt sich zum Beispiel allein auf das Kommunalwahlrecht. Fast komplett implementiert wurde die Idee einer europaweiten Gleichheit im Bereich des Arbeitsmarktes (vgl. Beck/Grande 2004: 170ff.). Geht man von der wohl nicht ganz unplausiblen Hypothese aus, dass der Arbeitsmarkt und die über die Erwerbstätigkeit auf dem Arbeitsmarkt erwirtschafteten Ressourcen einen der wichtigsten Bereiche des individuellen Lebens bildet, dann stellt die Veränderung der Zugangsmöglichkeiten zu einem Arbeitsmarkt einen zentralen, weil für die Lebensführung der Menschen folgenreichen Eingriff in die nationalstaatlich kasernierten Gleichheitsvorstellungen dar.

Die Veränderung der Zugangsmöglichkeiten zu den nationalen Arbeitsmärkten ist Bestandteil der Entwicklung eines europäischen Binnenmarktes. Unter europäischem Binnenmarkt wird der gemeinsame Markt der EU verstanden, in dem die Hindernisse für den freien Waren-, Kapital-, Personen- und Dienstleistungsverkehr beseitigt worden sind. Wie in der Einheitlichen Europäischen Akte von 1987 vorgesehen, begann zur Jahreswende 1992/1993 der gemeinsame europäische Binnenmarkt mit den sogenannten vier Freiheiten. Personen, Waren, Dienstleistungen und Kapital sollten sich in der EU genauso frei bewegen können, wie es bisher innerhalb der nationalen Volkswirtschaften der Fall gewesen war. Im Kontext der Schaffung eines gemeinsamen Binnenmarktes wurde auch die Freizügigkeit für Nichterwerbstätige wie Studenten und Rentner ausgedehnt; zudem wurden die gegenseitige Anerkennung von Berufsabschlüssen und die Übertragung der in einem anderen Mitgliedsland erworbenen sozialen Ansprüche vereinbart.

Für unsere Fragestellung ist die sogenannte Freizügigkeitsregel für Arbeitnehmer die entscheidende Regelung. Diese besagt, dass jeder abhängig Beschäftigte in einem anderen Mitgliedsland unter denselben Bedingungen wie ein Inländer eine Beschäftigung suchen und dieser nachgehen darf. Die Freizügigkeitsregel gilt analog auch für Selbstständige (Niederlassungsrecht).

Mit der Verabschiedung und Implementierung der Freizügigkeitsregel ist die Idee einer europäischen Gleichheit, im Sinne der Chancengleichheit auf dem Arbeitsmarkt, zum europaweiten Rechtsanspruch geworden und hat die Idee einer national begrenzten Gleichheit, die Inländer und europäische Ausländer ungleich behandelt, ersetzt. Der Terminus europaweit muss noch etwas genauer spezifiziert werden, da für die neu beigetretenen Länder Übergangszeiten vereinbart wurden. Portugal und Spanien sind der EU 1986 beigetreten, die volle Freizügigkeit wurde aber erst 1993 implementiert, da man starke Migrationsbewegungen von den ökonomisch schwächeren Beitrittsländern in die wohlhabenderen Kernländer erwartete und befürchtete. Diese blieben aus: Im Durchschnitt sind es weniger als zwei Prozent aller Arbeitskräfte, die in einem EU-Land aus einem anderen Mitgliedsland kommen (vgl. Werner 2001: 12; Kvist 2004: 305).

Auch für die zehn Länder, die im Mai 2004 der EU beigetreten sind, wurden Übergangsregeln erlassen, da einige der alten EU Länder, vor allem Deutschland und Österreich mit massiven Wanderungsbewegungen rechneten (vgl. Kvist 2004). Die Übergangsregeln sind (a) auf bestimmte Länder und (b) Personengruppen bezogen 
und (c) zeitlich gestaffelt. ${ }^{3}$ Die Übergangsregelungen gelten nicht für Malta und Zypern; Malteser und Zyprioten dürfen also unmittelbar von der Freizügigkeitsregel Gebrauch machen. Malta kann darüber hinaus eine Schutzklausel erlassen, falls es einen großen Zustrom von Arbeitskräften nach Malta befürchtet.

Die Übergangsregeln gelten nicht für den Umzug in einen anderen Mitgliedstaat, um dort zu studieren oder zu wohnen; sie gelten auch nicht für Selbstständige, sondern nur für einen Umzug in einen anderen Mitgliedstaat, um dort als abhängig Beschäftigter zu arbeiten. Eine Ausnahme bezüglich der Gruppe der Selbständigen ist für die beiden Mitgliedstaaten, die traditionell die meisten Bürger von Bewerberländern auf aufgenommen haben (Deutschland und Österreich) festgelegt worden. Unternehmen aus den künftigen Mitgliedstaaten, die Arbeitnehmer schicken wollen, um einen Auftrag für sie zu erledigen, z. B. um ein Gebäude zu errichten, fallen auch unter die Übergangsregelungen.

Die Übergangsregeln gelten bis zu sieben Jahre. In den ersten zwei Jahren nach der Erweiterung lassen die alten Mitgliedstaaten Arbeitnehmer aus den neuen Mitgliedstaaten nach Maßgabe ihrer eigenen Rechtsvorschriften und nicht nach Maßgabe der Gemeinschaftsvorschriften über die Freizügigkeit zu. Zwei Jahre nach der Erweiterung legt die Kommission einen Bericht über die Lage vor; die Mitgliedstaaten müssen erklären, welches Verfahren sie ab diesem Zeitpunkt anwenden wollen. Nach weiteren drei Jahren werden die Mitgliedstaaten erneut aufgefordert, ihren Arbeitsmarkt voll zu öffnen. Nur wenn sie nachweisen können, dass auf dem Arbeitsmarkt erhebliche Störungen bestehen oder zu entstehen drohen, können sie weiter Einschränkungen vorschreiben. In jedem Fall wird aber nach weiteren zwei Jahren die völlige Freizügigkeit hergestellt.

Auch wenn für die neuen EU-Länder Übergangsregeln vereinbart wurden, sind diese doch auf einen relativ kurzen Zeitraum befristet, zudem legitimationsbedürftig und auf bestimmte Länder und Personengruppen begrenzt. Nach relativ kurzer Zeit wird die Idee der Gleichheit aller Bürger also im gesamten EU-Raum Geltung besitzen. Weiterhin ist mit dem Recht auf freien Zugang zu vormals geschlossenen Arbeitsmärkten eine Vielzahl an Folgerechten verbunden; sie werden im Folgenden zitiert aus einem Papier der EU-Kommission - einfach nur aufgelistet, um zu zeigen, wie weitgreifend die Europäisierung des Gleichheitsgedankens ist. Die Freizügigkeitsregel bedeutet unter anderem auch: die Gleichbehandlung mit Inländern in Beschäftigungsfragen, Anspruch auf die gleichen Sozialleistungen und Steuervorteile wie Inländer, Gleichstellung in Wohnungsfragen (z. B. Beantragung von Sozialwohnungen) und Gleichbehandlung bei Sozialleistungen und Steuervorteilen. Die Familie des Arbeitnehmers ist unabhängig von ihrer Staatsangehörigkeit berechtigt, bei ihm zu wohnen. Die Familie des Arbeitnehmers ist berechtigt, Familienleistungen auf derselben Grundlage zu beziehen wie Inländer. Weiterhin ist mit der Freizügigkeitsregel die vollständige Koordinierung der sozialen Sicherheit impliziert. Dies be-

3 Einen guten Überblick über die getroffenen Maßnahmen findet man in dem Artikel von Jon Kvist (2004). 
deutet: Übertragung der Rentenansprüche und sonstiger Leistungen; die erworbenen Rechte müssen einem Arbeitnehmer erhalten bleiben, wenn er sich in einen anderen Mitgliedstaat begibt. Die in den einzelnen Ländern geleisteten Beiträge zur sozialen Sicherheit werden ohne Unterschied zusammengerechnet, um zu gewährleisten, dass der Arbeitnehmer stets gedeckt ist und er die Versicherung bei Bedarf unmittelbar in dem neuen Land in Anspruch nehmen kann.

Die aufgelisteten Regelungen zeigen, dass mit der Freizügigkeitsregel eine tief greifende Umcodierung des nationalstaatlich begrenzten Gleichheitsgrundsatzes verbunden und dass die Europäisierung der Chancengleichheitsidee nicht nur auf den Arbeitsmarkt begrenzt ist. ${ }^{4}$

\section{Die Akzeptanz der Marktöffnung durch die Bürger der Europäischen Union}

Unterstützen die EU-Bürger das Skript der EU-Institutionen von einer Gleichheit der Arbeitskräfte oder favorisieren sie ein Ungleichheitskonzept, das zwischen Inländern und Ausländern unterscheidet? Wird die institutionelle Europäisierung der Gleichheitsidee also durch eine mentalitätsmäßige Europäisierung unterstützt? Gerade in Demokratien ist die Frage der Übereinstimmung von Politiken mit den Präferenzen der Bürger eine wichtige Frage, da die Politik von der Zustimmung der Bürger strukturell abhängig ist.

4 Diese Neucodierung ist nicht völlig konfliktfrei eingeführt worden und hat vor allem mit der Osterweiterung der EU in einigen Ländern, so auch in Deutschland, zu Protesten geführt. Einige Akteure, vor allem die Gewerkschaften, befürchten ein Lohndumping zu Ungunsten der deutschen Arbeitnehmer und protestierten z. B. gegen folgende Ereignisse: a. Mit Ausnahme von Bau-, Reinigungs- und Innendekorationsgewerbe können Unternehmen mit Sitz in den neuen EU-Staaten ihre Dienste in Deutschland frei anbieten und Angestellte zu den in ihren Ländern üblichen Gehältern und Sozialabgaben in Deutschland arbeiten lassen. Die Einschränkung der Dienstleistungsfreiheit in Bau-, Reinigungs- und Innendekorationsgewerbe kann aber mit einem einfachen Trick umgangen werden: Die uneingeschränkte Niederlassungsfreiheit für Selbständige führt in Verbindung mit der Anfang 2004 in Kraft getretenen Reform der deutschen Handwerksordnung, die für viele Handwerksberufe die Zulassungsbeschränkungen aufgehoben hat, dazu, dass sich viele Handwerker aus den Beitrittsstaaten als Ein-Mann-Unternehmen in Deutschland niederlassen oder zusammen Unternehmen gründen, in denen jeder einzelne als Gesellschafter auftritt. Da Steuern und Sozialabgaben nicht in Deutschland, sondern im Herkunftsland abgeführt werden müssen, können die osteuropäischen Handwerker ihre Dienste sehr billig anbieten. b. Für besondere Proteste sorgt die vom ehemaligen EU-Binnenmarktkommissar Frits Bolkestein im Rahmen der LissabonStrategie vorgeschlagene Dienstleistungsrichtlinie. Diese sieht vor, dass Dienstleistungsunternehmen nur noch dem Recht und der Aufsicht des Staates unterliegen, in dem sie eingetragen sind (Herkunftslandprinzip), und zwar auch dann, wenn sie ihre Tätigkeit dauerhaft in anderen Ländern ausüben. Darüber hinaus sollen Unternehmen, die ihre Beschäftigten grenzüberschreitend einsetzen (entsenden), diese im Arbeitsland nicht mehr anmelden und keine Arbeitspapiere mehr bereithalten müssen. Besonders in Frankreich und Deutschland gibt es seitens der Politik großen Widerstand gegen den Richtlinienentwurf. 
Wir analysieren die Wertorientierungen der Bürger auf der Grundlage einer Sekundäranalyse des European Values Survey von 1999/2000. ${ }^{5}$ Die nationalen Stichproben sind mit mindestens 1.000 Befragten für das jeweilige Land repräsentativ. Befragt wurden Personen ab dem 18. Lebensjahr in Form einer mündlichen Befragung. Wir unterscheiden in unseren Analysen neben den verschiedenen Ländern vier Aggregatsgruppen von Ländern: alte Mitgliedsländer der EU (15), neue Mitgliedsländer, die seit dem 1. Mai 2004 Mitglieder der EU sind, zukünftige Mitgliedsländer (Bulgarien und Rumänien) und die Türkei.

In der Europäischen Wertestudie gibt es für den Bereich des Arbeitsmarktes, also des freien Personenverkehrs, einen Indikator, der die Einstellungen der Bürger zur Marktgleichheit der Bürger erfragt. Die entsprechende Frage lautet: »Sagen Sie mir bitte zur folgenden Aussage, ob Sie zustimmen oder nicht zustimmen: Wenn die Arbeitsplätze knapp sind, sollten die Arbeitgeber Deutsche (bzw. die jeweilige Nationalität) gegenüber Ausländern vorziehen. « Geantwortet werden konnte auf einer dreistufigen Skala mit »stimme $\mathrm{zu}$ «, »stimme nicht $\mathrm{zu}$ « und »weder noch«. Die Frage ist relativ restriktiv formuliert, da sie nach der Gleichheit in Krisenzeiten fragt. Eine Zustimmung bedeutet eine Zustimmung zur nationalstaatlichen Schließung der Gleichheitsidee und damit die Ablehnung der EU-Position. In Grafik 1 (folgende Seite) sind deshalb die Prozentangaben für die Kategorie »stimme nicht zu« für die Länder aufgeführt (vgl. auch Gerhards/Hölscher 2005: 149; Hölscher 2005).

Wie die Grafik zeigt, findet die Idee einer europaweiten Gleichheit in allen Ländergruppen keine mehrheitliche Zustimmung. Allerdings fällt das Niveau der Ablehnung recht unterschiedlich aus. Während sich in den alten Mitgliedsländern der EU knapp 40 \% für eine Gleichbehandlung von Inländern und Ausländern aussprechen, sind es in den Ländern der beiden Beitrittsrunden jeweils nur gut $10 \%$. Die Türkei liegt mit einem Drittel Zustimmung auf einem mittleren Niveau.

Innerhalb der vier Ländergruppen zeigen sich deutliche Unterschiede zwischen den Ländern. So findet sich in Schweden, den Niederlanden, Dänemark und Luxemburg eine Mehrheit für die Offenheit des Arbeitsmarktes. Nur knapp verfehlt wird die Mehrheit in Belgien und Estland. Letzteres ist auch der einzige Beitrittskandidat, der den Durchschnitt der alten EU-Mitglieder erreicht. Nicht einmal $5 \%$ Unterstützung für die europaweite Gleichheitsidee finden sich dagegen in Litauen, Polen und Malta.

5 Gute Informationen zum European Values Survey findet man zum einen unter derNetzseite http://www.europeanvalues.nl, zum anderen in Loek Halman u.a. (2001). Der Datensatz ist über das Zentralarchiv für empirische Sozialforschung in Köln unter der Nummer 3811 zu beziehen. 
Grafik 1: Einstellungen zur Gleichheit von inländischen und ausländischen Arbeitskräften: »Inländer sollten gegenüber Ausländern bevorzugt werden« (Ablehnung in \%)

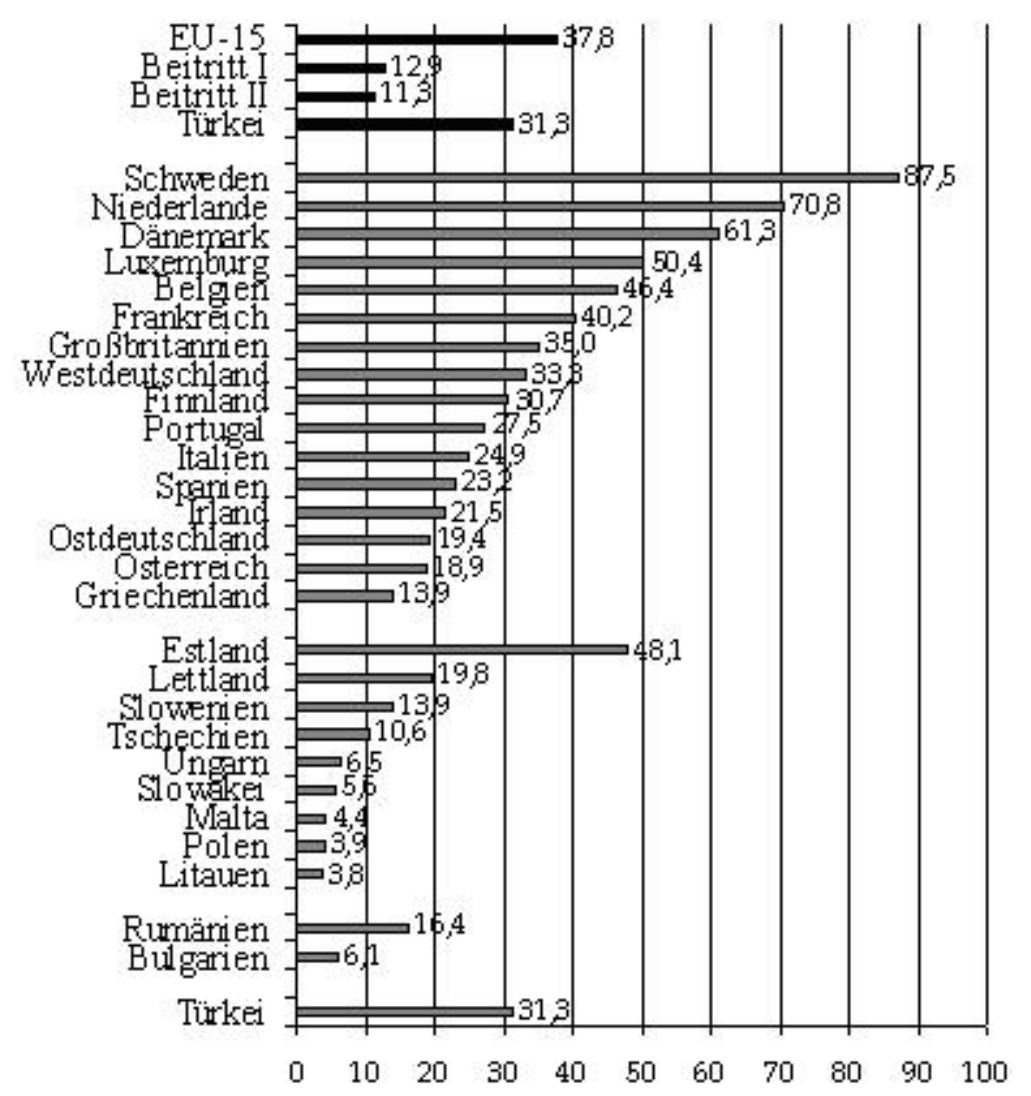

Quelle: European Values Survey 1999/2000

Nun bezieht sich der verwendete Indikator auf Ausländer insgesamt, die Marktoffenheit der EU-Position aber auf den europäischen Binnenmarkt. Es könnte sein, dass die Befragten mit dem Begriff Ausländer eher Personen von außerhalb der EU assoziieren, sich aber bei entsprechender Frageformulierung nicht gegen eine innereuropäische Öffnung des Arbeitsmarktes aussprechen würden. Glücklicherweise lässt sich diese Vermutung anhand einer Frage aus einem anderen Datensatz, nämlich dem Eurobarometer Nr. 53 aus dem Jahr 2000, zumindest ansatzweise überprüfen. ${ }^{6}$ Dort wurde gefragt, ob man verschiedene Personengruppen, die im jeweiligen Befragungsland arbeiten wollen, zulassen sollte oder nicht. Als Gruppen wurden Moslems, Personen aus Osteuropa, aus Krisengebieten, politisches Asyl Suchende sowie Personen aus anderen EU-Ländern genannt. Es zeigt sich, dass Personen, die das Gleichheitsprinzip unterstützen, dies für alle abgefragten Gruppen in relativ gleicher Weise tun. Bildet man einen additiven Index aus den verschiedenen Fragen, so ergibt sich ein erstaunlich hohes Cronbach's Alpha von 0,89 (vgl. Hölscher 2005). Man kann

6 Leider umfasst der Datensatz nicht alle hier analysierten Länder. Auf eine ausführlichere Präsentation der Daten wird daher verzichtet. 
also davon ausgehen, dass unser Indikator eine zuverlässige Messung für die EUPosition der Offenheit des Binnenmarktes ist.

Tabelle 1: Entwicklung der Einstellungen zur Gleichheit von inländischen und ausländischen Arbeitskräften: »Inländer sollten gegenüber Ausländern bevorzugt werden« (Ablehnung in \%)

\begin{tabular}{|c|c|c|c|c|}
\hline & 1990 & 1995/98 & 2000 & $\begin{array}{c}\text { Entwicklungs- } \\
\text { trend }\end{array}$ \\
\hline EU-15 & 29,7 & 35,4 & 33,9 & * \\
\hline Schweden & $56,7 \%$ & $74,3(1996)$ & $77,8 \%$ & + \\
\hline Niederlande & $62,0 \%$ & -- & $64,3 \%$ & * \\
\hline Dänemark & $37,9 \%$ & -- & $54,3 \%$ & + \\
\hline Luxemburg & -- & -- & $44,9 \%$ & \\
\hline Belgien & $26,9 \%$ & -- & $43,7 \%$ & + \\
\hline Frankreich & $31,2 \%$ & -- & $36,1 \%$ & $*$ \\
\hline Großbritannien & $41,5 \%$ & $39,1 \%(1998)$ & $31,6 \%$ & - \\
\hline Deutschland-West & $29,7 \%$ & $46,6 \%(1997)$ & $29,5 \%$ & - \\
\hline Finnland & $17,0 \%$ & $18,2 \%(1996)$ & $28,5 \%$ & + \\
\hline Portugal & $8,1 \%$ & -- & $24,5 \%$ & + \\
\hline Italien & $17,8 \%$ & -- & $20,3 \%$ & * \\
\hline Irland & $28,5 \%$ & -- & $20,2 \%$ & - \\
\hline Spanien & $16,6 \%$ & $12,6 \%(1996)$ & $19,6 \%$ & $*$ \\
\hline Österreich & $17,1 \%$ & -- & $17,1 \%$ & * \\
\hline Deutschland-Ost & $24,9 \%$ & $21,8 \%(1997)$ & $16,8 \%$ & - \\
\hline Griechenland & -- & -- & $12,7 \%$ & \\
\hline Beitritt I & 7,2 & 19,4 & 11,9 & * \\
\hline Estland & $3,0 \%$ & $40,0 \%(1996)$ & $43,9 \%$ & * \\
\hline Lettland & $4,9 \%$ & $41,4 \%(1996)$ & $18,3 \%$ & - \\
\hline Slowenien & $15,1 \%$ & 7,5\% (1995) & $12,2 \%$ & * \\
\hline Tschechien & $6,9 \%$ & -- & $9,8 \%$ & $*$ \\
\hline Ungarn & $10,9 \%$ & -- & $6,2 \%$ & * \\
\hline Slowakei & $5,2 \%$ & -- & $5,2 \%$ & * \\
\hline Malta & -- & -- & $4,3 \%$ & \\
\hline Litauen & $2,6 \%$ & 3,5\% (1996) & $3,7 \%$ & * \\
\hline Polen & $4,5 \%$ & 3,7\% (1996) & $3,7 \%$ & * \\
\hline Beitritt II & 12,4 & 6,6 & $10,2 \%$ & \\
\hline Bulgarien & $10,5 \%$ & 6,6\% (1998) & $5,8 \%$ & * \\
\hline Rumänien & $14,2 \%$ & -- & $14,6 \%$ & * \\
\hline Türkei & $21,5 \%$ & $17,2 \%(1996)$ & $30,8 \%$ & \\
\hline
\end{tabular}

Quelle: World Values Surveys 1990, 1995/98, 2000

Durch eine Messung zu einem Zeitpunkt lässt sich allerdings noch keine Aussage darüber treffen, ob sich nicht im Zeitverlauf die Gleichheitsvorstellungen der Bürger zunehmend europäisiert haben, wenn auch auf niedrigem Niveau. Da die Frage des European Values Survey in einigen europäischen Länden bereits 1990 und 1995 im Kontext des World Values Survey gestellt wurde, gibt es die Möglichkeit, zumindest ansatzweise zu prüfen, ob in dem Zeitraum, in dem gerade eine Öffnung der Ar- 
beitsmärkte in Europa durchgeführt wurde, auch ein Mentalitätswandel der Bevölkerung stattgefunden hat. Tabelle 1 gibt die Ergebnisse unserer Analysen wieder.

Wir setzen eine Veränderungsmarge von mindestens $5 \%$ an, um von einem Wechsel der Einstellungen der Bürger sprechen zu können. Betrachtet man die vier Aggregatskategorien, so zeigt sich, dass es in den EU-15-Ländern und der Türkei zu einer Zunahme der Akzeptanz europäischer Gleichheitsvorstellungen gekommen ist. Die Veränderungen innerhalb der EU-15-Länder beziehen sich aber auf eine Zeitphase, die vor der Einführung der Freizügigkeitsregel liegt; zwischen 1995 und 2000 bleiben die Werte hingegen konstant. In den beiden Beitrittsländern Bulgarien und Rumänien hat keine Veränderung stattgefunden, in den Ländern, die 2004 der EU beigetreten sind, gibt es einen leicht u-förmigen Verlauf. Ähnlich ambivalent und damit nicht eindeutig sind die Ergebnisse, wenn man sich die Entwicklungen in den einzelnen Ländern anschaut. In 14 Ländern gibt es keine Veränderung, in vier Ländern ist es zu einer Verstärkung der nationalen Schließung gekommen, in fünf Ländern zu einer Europäisierung der Gleichheitsvorstellung (Schweden, Finnland, Dänemark, Portugal und Belgien). Insgesamt bedeuten diese Befunde aber, dass es im Zeitverlauf nicht zu einer Europäisierung der Gleichheitsvorstellungen gekommen ist, die institutionelle Veränderung sich also nicht in den Mentalitäten der Bürger spiegelt. Dieses Ergebnis widerspricht damit der von Ulrich Beck und Edgar Grande (2004: 266) formulierten und in der Einleitung dieses Textes zitierten Hypothese.

\section{Erklärung der Einstellungen zur Europäisierung des Gleichheitsprinzips}

Wir werden in einem ersten Schritt Erklärungsfaktoren, die einen Einfluss auf die Einstellungen der Bürger zu einer Europäisierung der Gleichheitsidee haben können, diskutieren und dann empirisch prüfen, ob und in welcher Stärke die genannten Faktoren wirksam sind. ${ }^{7}$

1. Die von uns untersuchten Länder unterscheiden sich im Grad ihrer - vor allem ökonomischen - Modernität. Karl Marx war wahrscheinlich einer der ersten Autoren, der einen kausalen Zusammenhang zwischen den ökonomischen Lebensbedingungen einerseits und den Werten der Menschen andererseits unterstellt hat. Dieses abstrakte Kerntheorem liegt auch den meisten Modernisierungstheorien zugrunde. Es würde den Rahmen dieser Arbeit sprengen, die komplette Modernisierungstheorie mit ihren vielfältigen Facetten, die vielfach an der Modernisierungstheorie geäußerten Kritikpunkte und die darauf erfolgten Revisionen zu rekonstruieren (vgl. dazu die Überblicksartikel von Berger 1996; Inglehart 2001; Knöbl 2003; Zapf 1998). Wir wissen bis heute nicht genau, welche Faktoren eine Modernisierung befördert haben

7 Dabei berücksichtigen wir zum Teil andere erklärende Variablen als diejenigen, die wir in dem Gesamtprojekt analysiert haben (vgl. Gerhards/Hölscher 2005; Hölscher 2005). 
und wie die Kausalbeziehungen zwischen den verschiedenen Faktoren zu bestimmen sind ${ }^{8}$

Mit der durch den Modernisierungsprozess erfolgten Steigerung des ökonomischen Wohlstands geht aber eine Veränderung der Werteeinstellungen der Bürger einher. Die Zunahme der Möglichkeit zur Befriedigung materieller Bedürfnisse führt mit einer Zeitverzögerung, so die Inglehart'sche These des Wertewandels, zu einer Werteverschiebung von materialistischen hin zu postmaterialistischen Werten (1971; 1983; 1989; 1997). Materielle Werte sind Werte der Befriedigung der ökonomischen Lebensbedingungen und der Sicherheit, aber auch Werte der nationalen Identifikation und der nationalen Schließung und Ausgrenzung. Zu den postmateriellen Werten gehören unter anderem Wünsche nach Selbstentfaltung und Partizipation, aber auch Vorstellungen der Öffnung der nationalen Begrenzung und der Internationalität. Wir erwarten entsprechend, dass Befragte in ökonomisch geringer entwickelten Ländern sich in schwächerem Maße für eine Europäisierung der Gleichheitsvorstellung aussprechen als Personen aus Ländern, in denen ein höherer Grad an ökonomischer Modernität erreicht worden ist. Die von uns untersuchten Gesellschaften unterscheiden sich im Grad der ökonomischen Modernisierung und des damit verbundenen gesellschaftlichen Wohlstandes. Wir messen den Grad der ökonomischen Modernisierung eines Landes durch die Höhe des Human Development Index (HDI). In den HDI gehen drei Maßzahlen ein: Reales Bruttosozialprodukt pro Einwohner, Bildungsniveau und die durchschnittliche Lebenserwartung.

Der von uns analysierte Datensatz enthält aber auch eine unmittelbare Messung der materialistischen und postmaterialistischen Werteorientierung der Bürger, da die Befragung die Items enthält, aus denen der sogenannte Inglehart-Index gebildet werden kann. Wir haben neben der Makrovariable des Human Development Index also zusätzlich die Individualvariable des Materialismus/Postmaterialismus Index in der Analyse berücksichtigt. ${ }^{9}$ Dabei gehen wir von der Hypothese aus, dass Postmaterialisten eine Europäisierung der Gleichheitsidee eher unterstützen als Materialisten.

2. Die Öffnung von Grenzen und der Abbau nationaler Schließungen können zur Erhöhung der Konkurrenz vor allem auf dem Arbeitsmarkt führen. Je stärker fremde Arbeitskräfte als Bedrohung des eigenen Status interpretiert werden, desto eher

8 Das Ergebnis des Prozesses der Modernisierung ist aber ein historisch einmaliges Wachstum der Ökonomie und des Wohlstands der Bürger (Maddison 1995: 21). Wie auch immer man das Wachstum und die Wohlstandsentwicklung sich modernisierender Gesellschaften erklären kann, auf der Ebene der Phänomenbeschreibung gibt es eine weitgehende Übereinstimmung zwischen den verschiedenen Theoretikern dahingehend, dass modernisierte Gesellschaften durch ein Set von Merkmalen beschreibbar (nicht erklärbar) sind, die zusammen ein Syndrom bilden (vgl. Norris 2002: 20ff.; Bell 1979; 1996).

9 Der Inglehart-Index wurde auf der Grundlage folgender Fragen gebildet: »There is a lot of talk these days about what the aims of this country should be for the next ten years. Which of the things would you say is most/next most important: (1) Maintaining order in the nation, (2) Giving people more say in government decisions, (3) Fighting rising prices, (4) Protecting freedom of speech.« Daraus wurde eine neue Variable nach dem Inglehart-Index gebildet: 1 = Materialist, 2 = MischtypMaterialist, 3 = Mischtyp-Postmaterialist, 4 = Postmaterialist. 
werden sich Befragte für eine nationalstaatliche Schließung aussprechen. Diese Grundhypothese ist sehr früh in der sozialpsychologischen Vorurteilsforschung und in der Realistic Group Conflict Theory formuliert und getestet worden (Campbell 1965; Sherif 1966). Die Autoren gehen davon aus, »that group conflicts are rational in the sense that groups do have incompatible goals and are in competition for scarce resources«. (Campbell 1965: 287) Gruppeninteressen beziehen sich auf »a real or imagined threat to the safety of the group, an economic interest, a political advantage, a military consideration, prestige, or a number of others«. (Sherif 1966: 15) Feindseligkeiten, Konflikte, Aggressionen und negative Einstellungen wie Vorurteile resultieren demnach aus dem aktuellen Wettbewerb zwischen den Gruppen und aus der Konkurrenz um knappe Ressourcen. Befinden sich Gruppen in diesem von ihnen wahrgenommenen Zustand, dann kommt es zur Aktualisierung von Vorurteilen und zur Schließung gegenüber Fremdgruppen. Zahlreiche empirische Bestätigungen dieser und ähnlicher Ansätze finden sich in historischen Studien, die sich mit der Veränderung von Stereotypen und Vorurteilen in Abhängigkeit von Intergruppenkonflikten befassen (z. B. Haslam u.a. 1992; vgl. Brown 1995: 166-168) und in Studien, die sich mit Problemen interethnischer Beziehungen infolge von Einwanderung auseinandersetzen (USA: Bonacich 1972; 1979; Olzak 1992; Südafrika: vgl. Duckitt 1992: 99-102; Europa: vgl. Schäfer/Six 1978: 133f.; von Freyberg 1994: 129-166; Quillian 1995: 586-611). ${ }^{10}$

Wir vermuten, dass diejenigen Personen und Bevölkerungsgruppen, die durch mögliche Einwanderungen aus anderen Ländern Nachteile für ihre eigenen Chancen auf dem Arbeitsmarkt und für ihre ökonomische Position antizipieren, sich eher gegen die Idee einer europäischen Gleichheit aussprechen als diejenigen Personen, für die dies nicht der Fall ist oder die sogar von Einwanderern profitieren. Wir wissen aus der Einwanderungssoziologie, dass - auch wenn die Zuwanderung europäischer ausländischer Arbeitskräfte auf die jeweiligen nationalen Arbeitsmärkte insgesamt gering ist - vor allem niedrig qualifizierte Personen aus den ärmeren Ländern in die wohlhabenderen Länder immigrieren. Hier treten sie auf dem Arbeitsmarkt in Konkurrenz zu den ebenfalls niedrig qualifizierten inländischen Arbeitnehmern; die besser verdienenden Bürger dürften hingegen eher von der erhöhten Konkurrenz auf dem unteren Segment des Arbeitsmarktes profitieren, weil die Lohnkonkurrenz wahrscheinlich zu Preisnachlässen auf Waren und Dienstleistungen führt. Während z. B. die Berliner Besserverdienenden von den polnischen Handwerkern und Putzfrauen, die sich seit der Osterweiterung als Selbständige niederlassen dürfen, profitieren werden, stellt sich die Situation für die deutschen Handwerker und Bauarbei-

10 Neben Sherif verifizierten zudem andere Sozialpsychologen einzelne Hypothesen der Realistic Group Conflict Theory im Rahmen von Gruppenexperimenten (z. B. Diab 1970; Rabbie u.a. 1974). Auch Jan Skrobanek (2004: 302) verweist in seiner Arbeit darauf, dass die »Definition der Situation als Konflikt um Güter (...) Prozesse der Abgrenzung « dynamisiert. Die genannten Untersuchungen zeigen, dass »tatsächlicher oder vermeintlicher Wettbewerb mit einer anderen Gruppe zu den stärksten Einflussfaktoren (gehört), die vorurteilsvolles Verhalten gegenüber dieser und ihren Mitgliedern begünstigt.« (Schäfer/Six 1978: 167) 
ter ganz anders dar. Durch die billigen Arbeitskräfte erhöht sich für sie die Konkurrenz auf dem Arbeitsmarkt, was sich negativ auf ihre eigenen Einkommenschancen auswirken wird. Die Idee einer europäischen Gleichheit kann für diese mit handfesten materiellen Nachteilen verbunden sein. Dies kann sie bewegen, sich eher für eine nationale Schließung auszusprechen.

Wir haben drei Variablen in die multivariaten Analysen aufgenommen, die die unterschiedlichen Interessenslagen der Bürger messen sollen; die Variablen Arbeitslos/Nicht arbeitslos und das Bildungsniveau auf der Individualebene und die Arbeitslosenquote pro Land auf der Makroebene. Wir erwarten, dass Arbeitslose, Personen aus Ländern mit hoher Arbeitslosenquote und Personen mit niedriger Bildung sich eher gegen die europäische Gleichheitsidee aussprechen werden als andere Personen. ${ }^{11}$

3. Menschen folgen nicht nur ihren Interessen, sondern auch ihren Ideen und ideologischen Orientierungen (vgl. dazu den Überblick über Experimente in Fehr/Fischbacher 2002). Das Links/Rechts-Schema stellt ein abstraktes ideologisches Raster dar, mit dem die Bürger konkrete politische Themen interpretieren. Es erleichtert den Bürgern eine Orientierung und eine eigene Positionsbestimmung bezüglich einer Vielzahl unterschiedlicher konkreter politischer Themen. Mit den Polen rechts und links sind jeweils unterschiedliche gesellschaftliche Werte und politische Ideologien verbunden. Dieter Fuchs und Hans-Dieter Klingemann (1990) haben diese Vorstellungen auf der Basis einer Umfrage in drei Ländern empirisch rekonstruiert. Mit rechten Vorstellungen sind im stärkeren Maße Ideen der nationalen Identität, des Nationalismus, der Systemerhaltung und der nationalen Schließung verbunden, mit linken Vorstellungen im höheren Maße Ideen der Gleichheit und Solidarität, des Sozialismus und der Internationalität. Wir vermuten, dass die Präferenz der Befragten für eine nationale Gleichheitsvorstellung oder eine transnationale europäische Gleichheitsvorstellung durch das Links-Rechts-Schema beeinflusst wird. Wir erwarten, dass Personen, die einer linken Orientierung zuneigen, sich eher für eine europä-

11 Die Frage zur Messung von Arbeitslosigkeit lautete: »Are you yourself employed now or not? If yes: About how many hours a week? (If more than one job: only for the main job.) Has paid employment: 30 hours a week ore more (1), Less than 30 hours a week (2), Self employed (3); If no paid employment: Retired/pensioned (4), Housewife not otherwise employed (5), Student (6), Unemployed (7), Other (8).« Für die Datenauswertung wurde eine Dummyvariable mit den Merkmalsausprägungen $0=$ Erwerbstätig/Rentner/Hausfrau/Student/Andere und $1=$ Arbeitslos gebildet.

Die Bildungsvariable wurde durch folgende Frage gemessen: »What is the highest level you have reached in your education? To be measured as detailed as possible on national level and to be transformed into this variable: Inadequately completed elementary education (1), Completed (compulsory) elementary education (2), (Compulsory) elementary education and basic vocational qualification (3), Secondary, intermediate vocational qualification (4), Secondary, intermediate general qualification (5), Full secondary, maturity level certificate (6), Higher education - lower level tertiary certificate (7), Higher education - upper-level tertiary certificate (8).« 
ische Gleichheit, Personen, die sich im rechten Spektrum platzieren, sich eher für eine nationale Begrenzung des Gleichheitsgedankens einsetzen. ${ }^{12}$

Zur Überprüfung unserer Hypothesen haben wir drei verschiedene logistische Regressionen berechnet. In die erste Modellrechnung gehen allein die beiden Makrovariablen Human Development Index und Arbeitslosenquote ein. Der logistischen Regression wurde ein quadratischer Term des HDI hinzugefügt, da sich bei einer vorigen Analyse gezeigt hat, dass die Variable HDI einen nichtlinearen Effekt auf den Anteil der Bevölkerung hat, die sich für die europäische Gleichheitsidee aussprechen. In das zweite Modell gehen alle erläuterten Individualvariablen ein; zusätzlich haben wir das Alter der Befragten als Kontrollvariable berücksichtigt. Die dritte Modellrechnung schließlich berücksichtigt sowohl die Makro- als auch die Mikrovariablen.

Wie die Pseudo-R ${ }^{2}$-Werte in Tabelle 2 (folgende Seite) ausweisen, können wir die Einstellungen der Bürger zu einer Europäisierung der Gleichheitsvorstellung mit unseren theoretisch begründeten unabhängigen Variablen sehr gut erklären. Sowohl die beiden Makrofaktoren als auch die Mikrovariablen haben einen Einfluss auf die Gleichheitsvorstellungen der Bürger. Zusammengenommen können die beiden Variablen in dem integrierten dritten Modell $25 \%$ der Varianz erklären.

Die Vorzeichen der standardisierten Koeffizienten zeigen, dass alle unsere Hypothesen bestätigt werden. Je stärker ein Land modernisiert ist, aus dem der oder die Befragte kommt, desto höher ist die Wahrscheinlichkeit, dass er oder sie sich für eine Europäisierung der Gleichheitsidee ausspricht. Die Werteorientierung der Befragten hat darüber hinaus nochmals einen zusätzlichen Effekt auf dessen Gleichheitsvorstellung: Postmaterialisten sprechen sich im stärkeren Maße für die europäische Gleichheitsidee aus als Materialisten. Auch die Hypothese, dass die ideologische Orientierung des oder der Befragten einen Einfluss auf die Einstellungen zur Gleichheitsidee hat, wird durch die empirische Analyse bestätigt. Wie vermutet, scheint die Idee einer transnationalen Gleichheit im stärkeren Maße Teil einer linken Politikvorstellung zu sein, die Idee nationaler Schließung ist hingegen stärker in einer rechten Ideologie verankert.

12 Die Variable wurde durch folgende Frage gemessen: »In political matters, people talk of >the left and the right $<$. How would you place your views on this scale, generally speaking $(1=\operatorname{Left}$ to $10=$ Right). « Für die Datenanalyse wurde die Variable gespiegelt: $1=$ Rechts bis $10=$ Links. 
Tabelle 2: Erklärung der Einstellung zur Europäisierung der Gleichheitsvorstellung auf dem Arbeitsmarkt (binäre logistische Regressionen)

\begin{tabular}{|c|c|c|c|}
\hline & \multicolumn{3}{|c|}{$\begin{array}{c}\text { Zustimmung zur Gleichheit inländischer und } \\
\text { ausländischer Arbeitskräfte }{ }^{1}\end{array}$} \\
\hline & Modell 1 & Modell 2 & Modell 3 \\
\hline \multicolumn{4}{|l|}{ Makrovariablen } \\
\hline HDI & $\begin{array}{l}-18,478^{* * *} \\
(-5,94)\end{array}$ & & $\begin{array}{l}-19,260^{* * *} \\
(-5,74)\end{array}$ \\
\hline HDI-Quadrat & $\begin{array}{l}19,103^{* * *} \\
(5,98)\end{array}$ & & $\begin{array}{l}19,930^{* * *} \\
(5,78)\end{array}$ \\
\hline Arbeitslosenquote & $\begin{array}{l}-0,325^{* *} \\
(-2,67)\end{array}$ & & $\begin{array}{l}-0,405^{* * *} \\
(-3,71)\end{array}$ \\
\hline \multicolumn{4}{|l|}{ Mikrovariablen } \\
\hline Arbeitslos & & $\begin{array}{l}-0,074^{*} \\
(-1,99)\end{array}$ & $\begin{array}{l}-0,013 \\
(-1,02)\end{array}$ \\
\hline $\begin{array}{l}\text { Politische Orientierung } \\
\text { (Rechts-Links-Skala) }\end{array}$ & & $\begin{array}{l}0,213^{* *} \\
(3,04)\end{array}$ & $\begin{array}{l}0,216^{* *} \\
(2,93)\end{array}$ \\
\hline Bildung & & $\begin{array}{l}0,263^{* *} \\
(3,06)\end{array}$ & $\begin{array}{l}0,382^{* * *} \\
(5,96)\end{array}$ \\
\hline Alter & & $\begin{array}{l}-0,111^{* * *} \\
(-3,80)\end{array}$ & $\begin{array}{l}-0,095^{* *} \\
(-2,94)\end{array}$ \\
\hline $\begin{array}{l}\text { Inglehart- } \\
\text { Postmaterialismus-Index }\end{array}$ & & $\begin{array}{l}0,390^{* * *} \\
(6,57)\end{array}$ & $\begin{array}{l}0,340^{* * *} \\
(11,87)\end{array}$ \\
\hline $\begin{array}{l}\text { Pseudo R² (nach Nagel- } \\
\text { kerke) }\end{array}$ & 0,154 & 0,106 & 0,251 \\
\hline
\end{tabular}

Wir sind weiterhin davon ausgegangen, dass die Interessenlage der Befragten einen Effekt auf die Unterstützung einer Öffnung der Märkte für Arbeitskräfte hat. Arbeitslose und Personen im unteren Spektrum der Schichtpyramide der Sozialstruktur (hier gemessen durch den Grad der Bildung des/der Befragten) werden durch Zuwanderungen eher Nachteile in Kauf nehmen müssen, für Erwerbstätige und Personen mit hoher Bildung wird dies nicht oder weniger der Fall sein. Dies hat erwartbare Folgen für die Einstellungen der Bürger. Und in der Tat zeigt sich: Je geringer die Bildung des Befragten und je höher die Arbeitslosigkeit in einem Land, desto eher tendieren die Bürger zur nationalen Schließung und lehnen die europäische Gleichheitsidee ab. Das Vorzeichen des Regressionskoeffizienten für die individuelle Arbeitslosigkeit zeigt zwar in die richtige Richtung, der Wert ist aber nicht signifikant. Schließlich geht auch von der Kontrollvariable Alter ein Effekt in der erwarteten Richtung aus: Je jünger ein Befragter/eine Befragte, desto stärker fällt die Unterstützung für eine Europäisierung der Gleichheitsidee aus. 


\section{Zusammenfassung und Ausblick}

Im Mai 2005 haben die Bürger Frankreichs in einer Volksabstimmung den von den Regierungschefs der Mitgliedsländer der EU verabschiedeten Verfassungsentwurf abgelehnt. Einige Tage später sind die Niederländer in ihrer Volksabstimmung dem Votum der Franzosen gefolgt. In der nachfolgenden Debatte über die Motive der Ablehnung der Verfassung ist von politischen Beobachtern immer wieder betont worden, dass die Bürger nicht über die Verfassung, sondern über innenpolitische Probleme Frankreichs abgestimmt hätten. Dieser Eindruck wird durch eine nach dem Referendum durchgeführte Umfrage unterstützt. Kurz nach der Volksabstimmung hat die Europäische Kommission im Rahmen der Eurobarometer-Befragungen eine Sonderumfrage in Frankreich über die Motive der Ablehnung der Verfassung durchgeführt. Die folgende Tabelle gibt die Motive derjenigen wieder, die gegen die Verfassung gestimmt haben. Die Tabelle ist dem Eurobarometerbericht entnommen (European Commission 2005: 17).

Tabelle 3: Gründe, die diejenigen Franzosen genannt haben, die gegen die Verfassung gestimmt haben

\begin{tabular}{|c|c|}
\hline $\begin{array}{l}\text { What are all the reasons why you voted "No" at the referendum on the European } \\
\text { Constitution? }\end{array}$ & $\begin{array}{c}\text { Prozent } \\
\text { (Mehrfachnennungen } \\
\text { möglich) }\end{array}$ \\
\hline $\begin{array}{l}\text { It will have negative effects on the employment situation in France/relocation } \\
\text { of French enterprises/loss of jobs }\end{array}$ & 31 \\
\hline $\begin{array}{l}\text { The economic situation in France is too weak/there is too much unemploy- } \\
\text { ment in France }\end{array}$ & 26 \\
\hline Economically speaking, the draft is too liberal & 19 \\
\hline $\begin{array}{l}\text { Opposes the president of the Republic/the national government/certain politi- } \\
\text { cal parties }\end{array}$ & 18 \\
\hline Not enough social Europe & 16 \\
\hline Too complex & 12 \\
\hline Does not want Turkey in the European Union & 6 \\
\hline Loss of national sovereignty & 5 \\
\hline Lack of information & 5 \\
\hline I am against Europe/European construction/European integration & 4 \\
\hline I do not see what is positive in this text & 4 \\
\hline The draft goes too far/advances too quickly & 3 \\
\hline Opposition to further enlargement & 3 \\
\hline Not democratic enough & 3 \\
\hline Too technocratic/juridical/too much regulation & 2 \\
\hline I am against the Bolkestein directive & 2 \\
\hline $\begin{array}{l}\text { I do not want a European political union/a European federal State/the United } \\
\text { States of Europe }\end{array}$ & 2 \\
\hline The draft does not go far enough & 1 \\
\hline Other answers & 21 \\
\hline Don't know/No answer & 3 \\
\hline
\end{tabular}

Wie die Tabelle eindruckvoll ausweist, sind die mit Abstand wichtigsten Motive für die Ablehnung der Verfassung ökonomischer Natur; sie beziehen sich auf Fragen des 
Arbeitsmarktes und der Arbeitslosigkeit. Auch wenn die Inhalte der Verfassung mit Fragen des Arbeitsmarktes und der Arbeitslosigkeit recht wenig zu tun haben, hat der französische Souverän die seltene Gelegenheit einer unmittelbaren Einflussnahme auf das europäische Geschehen genutzt, um das zum Ausdruck zu bringen, was ihn bewegt und wofür er der EU die Verantwortung zuschreibt. Und die Ablehnungsmotive der Verfassung beziehen sich weitgehend auf die Fragen, die wir in diesem Artikel analysiert haben. Die Bürger scheinen die Verfassung mit der Freizügigkeitsregel in Verbindung zu bringen. Sie befürchten Nachteile für sich selbst und ihren nationalen Arbeitsmarkt. Wir vermuten, dass diese konkreten Einstellungen durch ein generalisiertes Muster der Interpretation von Gleichheit und Ungleichheit gesteuert werden. Fassen wir unsere Befunde diesbezüglich zusammen:

Wir haben auf der Basis der Auswertung der Daten des European Values Survey gesehen, dass die Bürger in Europa mehrheitlich und in erster Linie eine nationalstaatlich eingegrenzte Vorstellung von Gleichheit präferieren, die Vorstellung einer europäischen Gleichheit aller Marktbürger wird mehrheitlich abgelehnt. Allerdings fällt das Niveau der Ablehnung recht unterschiedlich aus. Während sich in den alten Mitgliedsländern der EU knapp 40 \% für offene Arbeitsmärkte aussprechen, sind es in den Ländern der beiden Beitrittsrunden jeweils nur gut $10 \%$, die Türkei liegt mit einem Drittel Zustimmung auf einem mittleren Niveau. Dies bedeutet aber, dass sich mit der Erweiterung der EU das Unterstützungsniveau für das EU-Gleichheitsskript deutlich abgesenkt hat bzw. mit der Aufnahme der neuen Länder weiter absenken wird. Zudem zeigen sich innerhalb der vier Ländergruppen deutliche Unterschiede. So findet sich in Schweden, den Niederlanden, Dänemark und Luxemburg eine Mehrheit für die Offenheit des Arbeitsmarktes. Nicht einmal $5 \%$ Unterstützung für die Offenheit des Marktes finden sich dagegen in Litauen, Polen und Malta. Die Tatsache, dass die Mehrheit der Europäer Inländer und europäische Ausländer auf dem Arbeitsmarkt ungleich interpretieren spricht sehr deutlich gegen eine Europäisierung sozialer Gleichheitsvorstellungen. Dies hat sich, wie der Vergleich von Befragungen zu drei verschiedenen Zeitpunkten gezeigt hat, auch im Zeitverlauf nicht verändert. Damit kollidieren die Vorstellungen der Bürger aber mit den in Recht gegossenen Vorstellungen der EU von einer Europäisierung der Gleichheit. Wir haben gesehen, dass die EU mit ihren Politiken eine nationalstaatlich begrenzte Codierung von Gleichheit ersetzt hat durch eine europäisch begrenzte Idee der Gleichheit, die alle Bürger Europas als Gleiche betrachtet und die legitimierte Ungleichheitsgrenze nach außen an die territorialen Grenzen der EU verschiebt.

Die Frage nach einer Unterstützung des EU-Skripts durch die Bürger ist für die Legitimität der europäischen Institutionen und ihrer Politiken aber nicht unerheblich. Ein mis-match zwischen Eliten-Projekt auf der einen Seite und den Meinungen der Bürger auf der anderen kann, wie die Ablehnung der europäischen Verfassung durch die Referenden in Frankreich und den Niederlanden gerade gezeigt haben, zu Legitimitätsproblemen der Institutionen insgesamt führen. Demokratische Institutionen sind von der Zustimmung ihrer Bürger abhängig. Fritz W. Scharpf $(1970 ; 2004)$ unterscheidet zwischen einer Input- und einer Output-Legitimation. Eine InputLegitimation von Institutionen setzt auf Seiten der Bürger eine kollektive Identität 
voraus, die auch die Zumutungen von Politiken, die den Interessen der Bürger widersprechen, abfängt, entsprechend belastungsresistent bzw. umverteilungsfest ist (Vobruba 1999). Dass es der Europäischen Union an einer gemeinsamen Identität mangelt, ist hinreichend bekannt; die Gründe dafür sind vielfältig und in der Literatur ausführlich diskutiert worden (vgl. Kielmannsegg 1996; Lepsius 1997; Gerhards 1993). Die Last, den Herrschaftsapparat der EU und den Integrationsprozess zu legitimieren, entfällt entsprechend auf Politiken, deren Effekte den Bürgern nutzen (Output-Orientierung). Und in der Tat scheint sich das Europäische Projekt der zunehmenden Integration in der Vergangenheit dadurch legitimiert zu haben, dass es (fast) allen, die daran partizipiert haben, genutzt hat. Es könnte nun sein, dass mit der Erweiterung der EU um eine Vielzahl an Ländern, die sich ökonomisch und kulturell zum Teil erheblich von den Kernländern der EU unterscheiden, ein Schwellenwert erreicht wurde, von dem ab die Konstellation einer win-win Situation nicht mehr gilt. Mögliche Abwanderungen aus den ökonomisch geringer entwickelten Beitrittsstaaten in die Wohlstandsländer werden als Bedrohung wahrgenommen. Die Grundlage der Interpretation dieser Situation als Bedrohung bildet ein Deutungsmuster, das einer nationalstaatlich begrenzten Codierung der Gleichheitsidee folgt. Wenn unsere Ursachenanalyse richtig ist, wird sich an den Einstellungen der Bürger auch kurzfristig nicht viel ändern, weil diese von recht langlebigen Strukturparametern wie dem Modernisierungsgrad einer Gesellschaft mitbestimmt werden. 


\section{Literatur}

Beck, Ulrich/Grande, Edgar (2004), Das kosmopolitische Europa, Frankfurt/M.

Bell, Daniel (1979), Die Zukunft der westlichen Welt. Kultur und Technologie im Widerstreit, Frankfurt/M.

Bell, Daniel (1996), Die nachindustrielle Gesellschaft, Frankfurt/M.

Berger, Johannes (1996), »Was bedeutet die Modernisierungstheorie wirklich und was wird ihr bloß unterstellt?«, Leviathan, Jg. 24, H. 1, S. 45-62.

Bonacich, Edna (1972), »A Theory of Ethnic Antagonism. The Split Labor Market«, American Sociological Review, Jg. 37, H. 5, S. 547-559.

Bonacich, Edna (1979), »The Past, Present, and Future of Split Labor Market Theory«, Research in Race and Ethnic Relations, Bd. 1, Amsterdam u.a., S. 17-64.

Brown, Rupert (1995), Prejudice. Its Social Psychology, Oxford.

Campbell, Donald Thomas (1965), »Ethnocentric and other altruistic motives«, in: David Levine (Hg.): Nebraska Symposium on Motivation, Lincoln, S. 283-311.

Delhey, Jan/Kohler, Ulrich (2005), Europäisierung sozialer Ungleichheit: Die Perspektive der Referenzgruppen-Forschung, Beitrag in diesem Band.

Diab, Lutfy N. (1970), »A Study of Intragroup and Intergroup Relations among Experimentally Produced Small Groups«, Genetic Psychology Monographs, Jg. 82, S. 49-82.

Duckitt, John (1992), The Social Psychology of Prejudice, New York.

European Commission (2005), The European Constitution: Post-referendum Survey in France. Flash Eurobarometer, (http://europa.eu.int/comm/public_opinion/flash/fl171_en.pdf; Stand: 14. Juli 2005)

Fehr, Ernst/Fischbacher, Urs (2002), »Why Social Preferences Matter - The impact of Non-selfish Motives on Competition, Cooperation and Incentives«, The Economic Journal, Jg. 112, C1-C33.

Fligstein, Neil/Stone Sweet, Alec (2002), »Constructing Polities and Markets. An Institutionalist Account of European Integration«, American Journal of Sociology, Jg. 107, S. 1206-1243.

Freyberg, Thomas von (1994), »Ausländerfeindlichkeit am Arbeitsplatz. Zur Untersuchung ethnischer Konflikte zwischen deutschen und ausländischen Beschäftigten«, in: Institut für Sozialforschung (Hg.), Rechtsextremismus und Fremdenfeindlichkeit: Studien zur aktuellen Entwicklung, Frankfurt am Main/New York, S. 129-166.

Fuchs, Dieter/Klingemann, Hans-Dieter (1989), »Das Links-Rechts-Schema als politischer Code: ein interkultureller Vergleich auf inhaltsanalytischer Grundlage«, in: Max Haller, Hans-Joachim Hoffmann-Nowotny und Wolfgang Zapf (Hg.), Kultur und Gesellschaft. Verhandlungen des 24. Deutschen Soziologentages, des 11. Österreichischen Soziologentages und des 8. Kongresses der Schweizerischen Gesellschaft für Soziologie in Zürich 1988, Frankfurt am Main/New York, S. 484-498.

Fuchs, Dieter/Klingemann Hans-Dieter (1990), »The Left-Right Schema«, in: M. Kent Jennings, Jan van Deth u.a., Continuities in Political Action - A Longitudinal Study of Political Orientations in Three Western Democracies, Berlin/New York, S. 203-234.

Gerhards, Jürgen (1993), »Westeuropäische Integration und die Schwierigkeiten der Entstehung einer europäischen Öffentlichkeit«, Zeitschrift für Soziologie, Jg. 22, H. 2, S. 96-110.

Gerhards, Jürgen/Hölscher, Michael (2003), »Kulturelle Unterschiede zwischen Mitglieds- und Beitrittsländern der EU. Das Beispiel Familien- und Gleichberechtigungsvorstellungen«, Zeitschrift für Soziologie, Jg. 32, H. 3, S. 206-225.

Gerhards, Jürgen, unter Mitarbeit von Michael Hölscher (2005), Kulturelle Unterschiede in der Europäischen Union. Ein Vergleich zwischen Mitgliedsländern, Beitrittskandidaten und der Türkei, Wiesbaden.

Halman, Loek (2001), The European Values Study: A Third Wave. Source Book of the 1999/2000 European Values Study Surveys, Tilburg.

Haslam, S. Alexander/Turner, John C./Oakes, Penelope J./McGarty, Craig/Hayes, Brett K. (1992), "Context-dependent Variation in Social Stereotyping 1: The Effects of Intergroup Relations as Mediated by Social Change and Frame of Reference«, European Journal of Social Psychology, Jg. 22, S. 3-20. 
Heidenreich, Martin (2005), Die Europäisierung sozialer Ungleichheit zwischen nationaler Solidarität, europäischer Koordinierung und globalem Wettbewerb, Einleitung in diesem Band.

Hölscher, Michael (2005), Wirtschaftskulturen in der erweiterten EU. Eine empirische Analyse der Einstellungen der Bürgerinnen und Bürger zur Ökonomie im europäischen Vergleich, Unveröffentlichte Dissertation, Freie Universität Berlin.

Inglehart, Ronald (1971), »The Silent Revolution in Europe«, American Political Science Review, Jg. 65, S. 991-1017.

Inglehart, Ronald (1983), »Traditionelle politische Trennungslinien und die Entwicklung der neuen Politik in westlichen Gesellschaften", Politische Vierteljahresschrift Jg. 24, S. 139-165.

Inglehart, Ronald (1989), Kultureller Umbruch, Frankfurt am Main/New York.

Inglehart, Ronald (1997), Modernization and Postmodernization. Cultural, Economic and Political Change in 43 Societies, Princeton.

Inglehart, Ronald (2001), »Sociological Theories of Modernization«, in: Neil J. Smelser und Paul B. Baltes (Hg.), International Encyclopedia of the Social and Behavioral Sciences, Amsterdam u.a., S. 99659971.

Kielmannsegg, Peter Graf (1996), »Integration und Demokratie« in: Markus Jachtenfuchs und Beate Kohler-Koch (Hg.), Europäische Integration, Opladen, S. 47-71.

Knill, Christoph (2001), The Europeanisation of National Administrations. Patterns of Institutional Change and Persistence, Cambridge.

Knöbl, Wolfgang (2003), »Theories that Won't Pass Away: The Never Ending Story of Modernization Theory «, in: Gerard Delanty und Engin F. Isin (Hg.), Handbook of Historical Sociology, London u.a., S. 96-107.

Konferenz der Vertreter der Regierungen der Mitgliedstaaten (2004), Vertrag über eine Verfassung für Europa, Brüssel, Stand: 25. Oktober 2004.

Kvist, Jon (2004), »Does EU enlargment start a race to the bottom? Strategic interaction among EU members states in social policy», Journal of European Social Policy, Jg. 14, S. 301-318.

Lepsius, Rainer M. (1990), »Der europäische Nationalstaat: Erbe oder Zukunft«, in: Rainer M. Lepsius, Interessen, Ideen, Institutionen, Opladen, S. 256-268.

Lepsius, Rainer M. (1997), »Bildet sich eine kulturelle Identität in der Europäischen Union?«, Blätter für deutsche und internationale Politik, Jg. 8, S. 948-955.

Maddison, Angus (1995), Monitoring the World Economy 1820-1992, Paris, Development Centre of the Organisation for Economic Co-operation and Development.

Märker, Alfredo (2001), »Zuwanderungspolitik in der Europäischen Union. Europäisierte Lösungen oder Politik des kleinsten gemeinsamen Nenners? «, Aus Politik und Zeitgeschichte, Jg. 2001, H. B8, S. 3-10.

Mau, Steffen, 2003, The Moral Economy of Welfare States. Britain and Germany compared. London und New York.

Müller-Graff, Peter-Christian (Hg.) (2000), Die Europäische Gemeinschaft in der Welthandelsorganisation. Globalisierung und Weltmarktrecht als Herausforderung für Europa, Schriftenreihe des Arbeitskreises Europäische Integration e.V., Bd. 47. Baden-Baden.

Norris, Pippa (2002), Democratic Phoenix, Cambridge.

Olzak, Susan (1992), The Dynamics of Ethnic Competition and Conflict, Stanford.

Quillian, Lincoln (1995), »Prejudice as a Response to Perceived Group Threat. Population Composition and Anti-immigrant and Racial Prejudice in Europe«, American Sociology Review, Jg. 60, S. 586611.

Rabbie, Jacob M./Benoist, Frits/Oosterbaan, Henk/Visser, Lieuwe (1974), »Differential Power and Cooperative Intergroup Interaction on Intragroup and Outgroup Attitudes«, Journal of Personality and Social Psychology, Jg. 30, S. 46-56.

Roller, Edeltraud (2000), »Marktwirtschaftliche und wohlfahrtsstaatliche Gerechtigkeitsprinzipien in Deutschland und den USA», in Jürgen Gerhards (Hg.), Die Vermessung kultureller Unterschiede. Opladen, S. 89-110. 
Schäfer, Armin (2002), Vier Perspektiven zur Entstehung und Entwicklung der »Europäischen Beschäftigungspolitik «, MPIFG Discussion Paper 9/02, Köln.

Schäfer, Bernd/Six, Bernd (1978), Sozialpsychologie des Vorurteils, Stuttgart.

Scharpf, Fritz W. (1970), Demokratietheorie zwischen Utopie und Anpassung, Kronberg.

Scharpf, Fritz W. (2004), Legitimationskonzepte jenseits des Nationalstaats, MPIFG Working Paper 04/6, Köln.

Sherif, Muzafer (1966), Group Conflict and Co-operation. Their Social Psychology, London.

Skrobanek, Jan (2004): Regionale Identifikation, negative Stereotypisierung und Eigengruppenbevorzugung. Das Beispiel Sachsen, Wiesbaden.

Smith, Anthony D. (1983), »Nationalism and Social Theory«, British Journal of Sociology Jg. 34, S. 19-38.

Stone Sweet, Alec/Sandtholtz, Wayne/Fligstein, Neil (Hg.) (2001), The Institutionalization of Europe, Oxford.

Svallfors, Stefan (1997), »Worlds of Welfare and Attitudes to Redistribution: A Comparison of Eight Western Nations», European Sociological Review, Jg. 13, S. 283-304.

Thiel, Elke (1996), Die Europäische Union, München.

Vobruba, Georg (1999), »Währungsunion, Sozialpolitik und das Problem einer umverteilungsfesten Europäischen Identität«, Leviathan, Jg. 1, S. 78-94.

Vobruba, Georg (2005), Die Dynamik Europas, Wiesbaden.

Werner, Heinz (2001), »Wirtschaftliche Integration und Arbeitskräftewanderungen in der EU«, Aus Politik und Zeitgeschichte, Jg. 2001, H. B8, S. 11-19.

Wessels, Wolfgang (1997), »An Ever Closer Fusion? A Dynamic Macropolitical View on Integration Processes", Journal of Common Market Studies, Jg. 35, S. 267-299.

Zapf, Wolfgang (1998), »Modernisierung und Transformation«, in: Bernhard Schäfers und Wolfgang Zapf (Hg.), Handwörterbuch zur Gesellschaft der Bundesrepublik, Opladen, S. 472-482.

\section{Datensätze}

European Commission, Hartung, H., EUROBAROMETER 53 - Racism, Information Society, General Services, and Food Labeling, April-May 2000 [Computer file], distributes as ZA No. 3296 (Zentralarchiv für empirische Sozialforschung (ZA) Köln).

European Values Study, EUROPEAN VALUES STUDY, 1999/2000 [Computer file] 2003 / Release 1, The Netherlands, Germany: Tilburg University, Zentralarchiv für Empirische Sozialforschung, Cologne (ZA), Netherlands Institute for Scientific Information Services (NIWI), Amsterdam [producer], 2003. Germany: ZA Köln) [distributor], 2003.

World Values Study Group. WORLD VALUES SURVEY, 1995-1997 [Computer file]. ICPSR version. Ann Arbor, MI: Institute for Social Research [producer], 1998. Ann Arbor, MI: Inter-university Consortium for Political und Social Research [distributor], 1998. 